\title{
Bipolar Comparison of 3D Ear Models
}

\author{
Guy De Tré ${ }^{1}$, Dirk Vandermeulen ${ }^{2}$, Jeroen Hermans ${ }^{2}$, Peter Claeys ${ }^{2}$, \\ Joachim Nielandt ${ }^{1}$, and Antoon Bronselaer ${ }^{1}$ \\ 1 Department of Telecommunications and Information Processing, \\ Ghent University, Sint-Pietersnieuwstraat 41, B-9000 Ghent, Belgium \\ \{Guy.DeTre, Joachim. Nielandt, Antoon. Bronselaer\} @UGent . be \\ 2 Department of Electrical Engineering (ESAT), \\ KU Leuven, Kasteelpark Arenberg 10, box 2440, B-3001 Leuven, Belgium \\ \{dirk. vandermeulen, peter.claes\}@esat.kuleuven. be, \\ jeroen.hermans@uzleuven. be
}

\begin{abstract}
Comparing ear photographs is considered to be an important aspect of victim identification. In this paper we study how automated ear comparison can be improved with soft computing techniques. More specifically we describe and illustrate how bipolar data modelling techniques can be used for handling data imperfections more adequately. In order to minimise rescaling and reorientation problems, we start with $3 \mathrm{D}$ ear models that are obtained from 2D ear photographs. To compare two 3D models, we compute and aggregate the similarities between corresponding points. Hereby, a novel bipolar similarity measure is proposed. This measure is based on Euclidian distance, but explicitly deals with hesitation caused by bad data quality. Comparison results are expressed using bipolar satisfaction degrees which, compared to traditional approaches, provide a semantically richer description of the extent to which two ear photographs match.
\end{abstract}

Keywords: Ear comparison, data quality, bipolarity, similarity.

\section{Introduction}

Ear biometrics are considered to be a reliable source for disaster victim identification. Indeed, ears are relatively immune to variation due to ageing [9] and the external ear anatomy constitutes unique characteristic features [13. Moreover, ears are often among the intact parts of found bodies, automated comparison of photographs is in general faster and cheaper than DNA analysis and collecting ante mortem photographs is considered to be a humane process for relatives.

Although there is currently no hard evidence that ears are unique, there is neither evidence that they are not. Experiments comparing over ten thousand ears revealed that no two ears were indistinguishable [135] and another study revealed that fraternal and identical twins have a similar but still clearly distinguishable ear structure. More research is needed to examine the validity of uniqueness but, despite of that a match or mismatch of ear biometrics can provide forensic experts with useful information in identification tasks. This makes research on the comparison of ear photographs relevant and interesting. 
When considering a missing person and the found body of a victim, ear identification practically boils down to a comparison of a set of ear photographs of the missing person with a set of ear photographs of the victim. Ear pictures of a victim are taken in post mortem conditions and hence referred to as post mortem (PM) pictures. Pictures of a missing person are always taken ante mortem and therefore called ante mortem (AM) pictures. PM pictures are assumed to be of good quality because they are usually taken by forensic experts under controlled conditions: high resolution, correct angle, uniform lighting, with the ear completely exposed. AM photos are often of lower, unprofessional quality. They are not taken with the purpose of ear identification and in most cases are provided by relatives or social media. Because we have no control over the conditions in which these pictures were taken, we can only hope to retrieve the best we can. Moreover, parts of the ear might be obscured by hair, headgear or other objects. The ear can also be deformed by glasses, earrings or piercings. Efficiently coping with all these aspects that have a negative impact on the data quality and hence also on the comparison is a research challenge and the subject of this work.

A considerable part of related work focusses on comparisons where an ear photo from a given set of photos is compared to all photos in this set (e.g, [25 12 21] ). This is a simplified case because matches between identical photos are searched for. The work in this paper is more general because it involves the matching of identical ears on different photos. An important step of each automated ear comparison process is the ear recognition step during which corresponding extracted features from two ears are compared in order to decide whether the ears match or not. Related work on ear recognition can be categorised based on the feature extraction scheme used. Intensity based methods use techniques like principal component analysis, independent component analysis and linear discriminant analysis for the comparison (e.g., [26 22]). Other categories of methods are based on force field transformations (e.g., [3]), 2D ear curves geometry (e.g., [8]), Fourier descriptors [1], wavelet transformation (e.g., [1]), Gabor filters (e.g., [18) or scale-invariant feature transformation (e.g., [15]). A last category of comparison techniques are based on 3D shape features. Most approaches use an iterative closest point algorithm for ear recognition (e.g., [7 23 14 6 ]). In [24] both point-to-point and point-to-surface matching schemes are used, whereas the method in [20] is based on the extraction and comparison of a compact biometric signature. An elaborate survey on ear recognition is 2 .

Current approaches for ear recognition cannot adequately handle, measure and reflect data quality issues. Nevertheless, efficiently coping with aspects that have a negative impact on correct ear detection and ear recognition is recognised to be an important research challenge. Ear identification methods should not only support the annotation of areas of bad data quality in an ear photo, but also be able to quantify these and reflect their impact on the results of ear comparisons. Indeed, forensic experts would benefit from extra information expressing the quality of data on which comparisons are based. For example, the case where AM photo $A$ and PM photo $P$ only partially match, but both having sufficient quality, clearly differs from the case where $A$ and $P$ partially match but $A$ is of 
low quality. In this work we investigate if and how soft computing techniques can be used to explicitly cope with AM data of bad quality in ear recognition. We use a 3D ear model on which we apply a point-to-point comparison method. Bipolar data modelling techniques are applied to denote and quantify areas of bad data quality. A novel bipolar similarity measure is proposed and used for the comparison. Ear comparison results are expressed using bipolar satisfaction degrees 16. which quantify hesitation about each result caused by bad data quality of the AM photos.

The remainder of the paper is structured as follows. In Section 2 some preliminaries are given. Some general issues on bipolarity in ear comparison are explained. Next, some basic concepts and definitions of bipolar satisfaction degrees are described. In Section 3 the 3D ear model is described. Section 4 deals with ear recognition and comprises the main contribution of the paper. It consecutively describes how corresponding points in two ear models can be compared, proposes a novel bipolar similarity measure, describes how this bipolar similarity measure can be used for the comparison of two 3D ear models and discusses the interpretation of comparison results in a bipolar setting. Some conclusions and plans for related research are reported in Section 5 .

\section{Preliminaries}

\subsection{Bipolarity Issues in Ear Comparison}

In the context of information handling the term bipolarity is, among others, used to denote that information can be of a positive or negative nature [16. Positive information describes what is true, correct, preferred. Oppositely, negative information describes what is false, incorrect, not preferred. In most situations, especially in a scientific context, positive and negative information complement each other. This is called symmetric bipolarity [10. Boolean logic and probability theory are examples of mathematical frameworks where symmetric bipolarity is assumed. So-called dual bipolarity is assumed in possibility theory where positive and negative information are dually related to each other and measured on different scales based on the same source of knowledge. The most general form of bipolarity is heterogeneous bipolarity. Two separate knowledge sources provide positive and negative information which are independent and hence do not have to complement each other. In the remainder of the paper, heterogeneous bipolarity is assumed.

Victim identification by ear biometrics can be seen as a pattern recognition process where PM ear photos of a victim are reduced to a set of features that is subsequently compared with the feature sets that are obtained from the AM photos of missing persons in order to help determine the identity of the victim on the basis of the best match. The following steps are hereby distinguished:

1. Ear detection. Hereby, ears are positioned and extracted from the photos.

2. Ear normalisation and enhancement. Detected ears are transformed to a consistent ear model using, e.g., geometrical and photometric corrections. 
3. Feature extraction. Representative features are extracted from the ear model.

4. Ear recognition. Feature sets of AM and PM ears are compared. A matching score indicating the similarity between the ears is computed.

5. Decision. The matching scores are ranked and used to render an answer that supports forensic experts in their decision making.

Errors in the first three steps can undermine the utility of the process. So, features that are obtained from bad quality data should be handled with care. For that reason, we consider that a feature set provides us with heterogeneous bipolar information: some features are obtained from reliable data and positively contribute in the identification process, other features might turn out to be unreliable and might have a negative impact which should be avoided, while for still other features there can be hesitation about whether they are useful or not.

\subsection{Bipolar Satisfaction Degrees}

To efficiently handle heterogeneous bipolarity in the comparison process, bipolar satisfaction degrees are used [16. A bipolar satisfaction degree (BSD) is a couple

$$
(s, d) \in[0,1]^{2}
$$

where $s$ is the satisfaction degree and $d$ is the dissatisfaction degree. Both $s$ and $d$ take their values in the unit interval $[0,1]$ reflecting to what extent the BSD represents satisfied, resp. dissatisfied. The extreme values are 0 ('not at all'), and 1 ('fully'). The values $s$ and $d$ are independent of each other. A BSD can be used to express the result of a comparison in which case $s$ (resp. $d$ ) denotes to which extent the comparison condition is accomplished (resp. not accomplished).

Three cases are distinguished:

1. If $s+d=1$, then the BSD is fully specified. This situation corresponds to traditional involutive reasoning.

2. If $s+d<1$, then the BSD is underspecified. In this case, the difference $h=$ $1-s-d$ reflects the hesitation about the accomplishment of the comparison. This situation corresponds to membership and non-membership degrees in intuitionistic fuzzy sets 4 .

3. If $s+d>1$, then the BSD is overspecified. In this case, the difference $c=s+d-1$ reflects the conflict in the comparison results.

With the understanding that $i$ denotes a t-norm (e.g., min) and $u$ denotes its associated t-conorm (e.g., max), the basic operations for BSDs $\left(s_{1}, d_{1}\right)$ and $\left(s_{2}, d_{2}\right)$ are [16]:

- Conjunction. $\left(s_{1}, d_{1}\right) \wedge\left(s_{2}, d_{2}\right)=\left(i\left(s_{1}, s_{2}\right), u\left(d_{1}, d_{2}\right)\right)$

- Disjunction. $\left(s_{1}, d_{1}\right) \vee\left(s_{2}, d_{2}\right)=\left(u\left(s_{1}, s_{2}\right), i\left(d_{1}, d_{2}\right)\right)$

- Negation. $\neg\left(s_{1}, d_{1}\right)=\left(d_{1}, s_{1}\right)$. 


\section{3D Ear Model}

In our previous work, we used 2D ear images for accomplishing ear recognition [19]. Imperfect geometrical and photometric transformations of 2D AM photos put a limit on the quality of the results. To improve this approach we now use a 3D ear model. This 3D ear model is obtained by estimating the parameters of a mathematical shape function such that the resulting shape optimally fits the images of the ear. For a PM ear, a 3D camera image can be used, whereas for an AM ear usually a set of $2 \mathrm{D}$ photos is used. The description of this fitting process is outside the scope of this paper. At this point it is sufficient to assume that for each ear we obtained a 3D model that captures the three dimensional details of the ear surface as shown in Fig. 1 (left and middle).

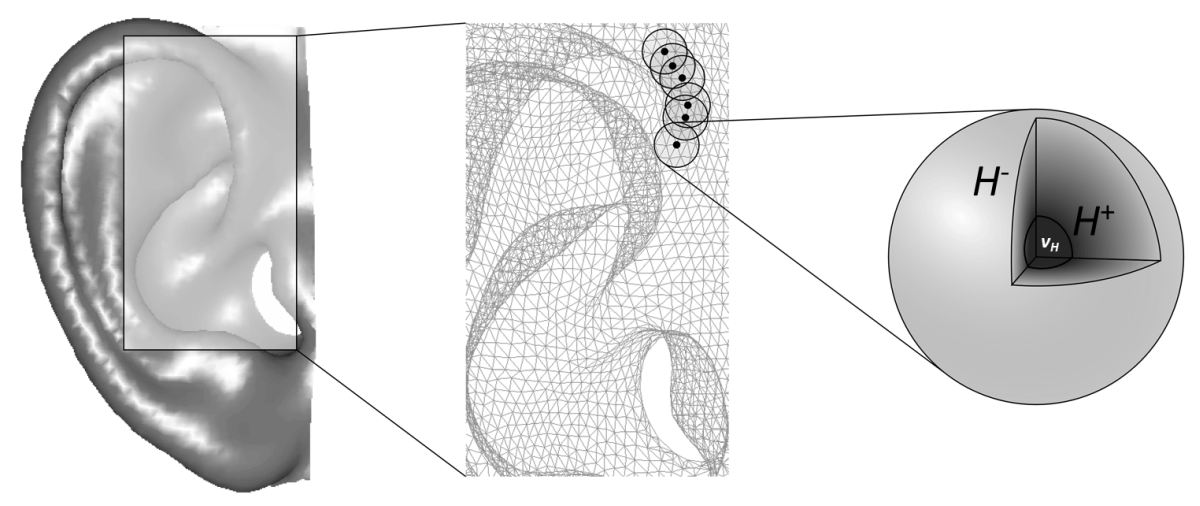

Fig. 1. 3D ear model (with hesitation spheres)

The 3D ear model is normalised for all ears, so all ear models have the same resolution and scale. However, unclear parts of 2D AM ear photos might decrease the quality of (parts of) a 3D AM ear model. Indeed, if parts of the 2D ears are inadequately visible or unreliable then their corresponding parts in the 3D model will as well be unreliable. To cope with this, unreliable parts of 3D ear models are indicated by so-called hesitation spheres. As illustrated in Fig. 1 (right), a hesitation sphere $H$ is defined by two concentric spheres $H^{+}$and $H^{-}$. All points $p$ inside or on the surface of the inner sphere $H^{+}$have a fixed associated hesitation value $\left.\left.h_{H}(p)=v_{H} \in\right] 0,1\right]$. For points on the surface or outside the outer sphere $H^{-}$the hesitation is 0 , whereas for points between both spheres the hesitation is gradually decreasing from $v_{H}$ to 0 , depending on their distance from $H^{+}$, i.e.,

$$
h_{H}(p)=v_{H} \cdot\left(1-\frac{d\left(H^{+}, p\right)}{d\left(H^{+}, H^{-}\right)}\right)
$$

where $d$ denotes the Euclidean distance. In general, forensic experts can manually assign as many hesitation spheres as required to indicate unreliable parts in the 
3D model. This assignment process is subject for (semi-)automation in future work. In the presence of $k>1$ hesitation spheres $H_{k}$, the overall hesitation about the quality of a point $p$ is computed by

$$
h(p)=\max _{k} h_{H_{k}}(p) .
$$

Thus, the maximal hesitation assigned to the point is taken.

Feature extraction boils down to selecting $n$ representative points of the $3 \mathrm{D}$ ear model. The more points that are considered, the better the matching results, but also the longer the computation time. For normalisation purposes, a fixed list $L_{S}=\left[p_{1}^{S}, \ldots, p_{n}^{S}\right]$ of $n$ points is selected on a standard, reference ear model $S$. Ear fitting, i.e., determining the optimal parameters for the shape function, will transform $L_{S}$ into a list $L_{E}=\left[p_{1}^{E}, \ldots, p_{n}^{E}\right]$ of $n$ points of the best fitting 3D ear model $E$. Hereby, each point $p_{i}^{S}$ corresponds to the point $p_{i}^{E}(i=1, \ldots, n)$. Moreover, using the same ear model $S$ and the same list $L_{S}$ for fitting two different ear models $A$ and $P$ guarantees that each point $p_{i}^{A}$ of $L_{A}$ corresponds to the point $p_{i}^{P}$ of $L_{P}(i=1, \ldots, n)$.

\section{Ear Recognition}

A basic step in ear recognition is the comparison of two left (or two right) ears. As such, in victim identification a set of AM photos of one ear have to be compared with a set of PM photos of the other ear. Using the 3D ear modelling technique explained in the previous section, the feature list $L_{A}$ of the ear model $A$ of the AM photos has to be compared with the feature list $L_{P}$ of the ear model $P$ of the PM photos. To reflect data quality issues, the hesitation $h(p)$ of each point in the lists $L_{A}$ and $L_{P}$ has to be taken into account.

\subsection{Similarity of Corresponding Features}

A commonly used comparison technique for corresponding points of two feature lists is to use the Euclidean distance. In the 3D space defined by the three orthogonal $X, Y$ and $Z$-axes, the Euclidean distance between a point $p^{A}$ of $L_{A}$ and its corresponding point $p^{P}$ in $L_{P}$ is given by:

$$
d\left(p^{A}, p^{P}\right)=\sqrt{\left(\left(p^{A}\right)_{x}-\left(p^{P}\right)_{x}\right)^{2}+\left(\left(p^{A}\right)_{y}-\left(p^{P}\right)_{y}\right)^{2}+\left(\left(p^{A}\right)_{z}-\left(p^{P}\right)_{z}\right)^{2}}
$$

where $(.)_{x},(.)_{y}$ and $(.)_{z}$ denote the $x, y$ and $z$ coordinates of the point.

The similarity between the points is then obtained by applying a similarity function to their distance. This similarity function $\mu_{\text {Sim }}$ can generally be defined by a fuzzy set Sim over the domain of distances, e.g.,

$$
\begin{aligned}
\mu_{\text {Sim }}:[0,+\infty[ & \rightarrow[0,1] \\
d & \mapsto 1, \text { iff } d \leq \epsilon_{1} \\
d & \mapsto 0, \text { iff } d \geq \epsilon_{0} \\
d & \mapsto 1-\frac{d-\epsilon_{1}}{\epsilon_{0}-\epsilon_{1}}, \text { iff } \epsilon_{1}<d<\epsilon_{0}
\end{aligned}
$$


where $0 \leq \epsilon_{1} \leq \epsilon_{0}$. Hence, if the distance $d<\epsilon_{1}$ then the similarity between the points is considered to be 1 , if $d>\epsilon_{0}$, the similarity is 0 , and for distances $d$ between $\epsilon_{1}$ and $\epsilon_{0}$ the similarity is gradually decreasing from 1 to 0 .

Hence the similarity between two points $p^{A}$ and $p^{P}$ yields

$$
\mu_{\text {Sim }}\left(d\left(p^{A}, p^{P}\right)\right) \in[0,1] .
$$

\subsection{Bipolar Similarity}

The similarity function $\mu_{\text {Sim }}$ is not taking into account any hesitation that might exist about the points $p^{A}$ and $p^{P}$. For that reason, the following novel similarity measure, based on both $\mu_{\text {Sim }}$ and the overall hesitation $h$ (cf. Eq. (3)), is proposed.

$$
\begin{aligned}
f_{\text {Bsim }}: \mathbb{P} \times \mathbb{P} & \rightarrow[0,1]^{2} \\
\left(p^{A}, p^{P}\right) & \mapsto(s, d)
\end{aligned}
$$

where $\mathbb{P} \subseteq \mathbb{R}^{3}$ denotes the $3 \mathrm{D}$ space in which the ear model is defined and $(s, d)$ is the BSD expressing the result of comparing $p^{A}$ and $p^{P}$ as described in the preliminaries. The $\mathrm{BSD}(s, d)$ is defined by

$$
s=\left(1-\max \left(h\left(p^{A}\right), h\left(p^{P}\right)\right)\right) \cdot \mu_{\operatorname{Sim}}\left(d\left(p^{A}, p^{P}\right)\right)
$$

and

$$
d=\left(1-\max \left(h\left(p^{A}\right), h\left(p^{P}\right)\right)\right) \cdot\left(1-\mu_{\operatorname{Sim}}\left(d\left(p^{A}, p^{P}\right)\right)\right) .
$$

Herewith it is reflected that we consider a consistent situation where $h=1-s-d$ and we consider $s$ (resp. $d$ ) to be the proportion of $1-h$ that corresponds with the similarity (resp. dissimilarity) between $p^{A}$ and $p^{B}$.

Remark that, with the former equations, the hesitation $h$ represented by the $\operatorname{BSD}(s, d)$ becomes $h=1-s-d=\max \left(h\left(p^{A}\right), h\left(p^{P}\right)\right) \in[0,1]$. Also $s+d=$ $1-h \leq 1$, such that the resulting BSD can either be fully specified, or be underspecified. So, Eq. 7 is consistent with the semantics of BSDs.

\subsection{Comparing 3D Ear Models}

The comparison of an AM ear model $A$ and a PM ear model $P$ is based on the comparison of all features $p_{i}^{A}$ and $p_{i}^{P}$ in their respective feature lists $L_{A}$ and $L_{P}$ $(i=1, \ldots, n)$. More specifically, the comparison results of all $n$ corresponding $\mathrm{AM}$ and PM points should be aggregated to an overall similarity and hesitation indication. Because these overall similarity and hesitation have to reflect the global similarity and hesitation of all points under consideration, the arithmetic mean can be used as an aggregator for the $n$ similarities of the corresponding points in the feature lists. Therefore we propose the following similarity measure for feature lists of $3 \mathrm{D}$ ear models.

$$
\begin{aligned}
f_{\text {bsim }}^{*}: \mathbb{P}^{n} \times \mathbb{P}^{n} & \rightarrow[0,1]^{2} \\
\left(\left[p_{1}^{A}, \ldots, p_{n}^{A}\right],\left[p_{1}^{P}, \ldots, p_{n}^{P}\right]\right) & \mapsto(s, d)
\end{aligned}
$$


where $\mathbb{P}^{n}$ denotes the set of all feature lists consisting of $n$ points of $\mathbb{P}$ and $(s, d)$ expresses the result of comparing the feature lists $L_{A}=\left[p_{1}^{A}, \ldots, p_{n}^{A}\right]$ and $L_{P}=\left[p_{1}^{P}, \ldots, p_{n}^{P}\right]$. The $\operatorname{BSD}(s, d)$ is defined by

$$
s=\left(1-\frac{\sum_{i=1}^{n} \max \left(h\left(p_{i}^{A}\right), h\left(p_{i}^{P}\right)\right)}{n}\right) \cdot \frac{\sum_{i=1}^{n} \mu_{\operatorname{Sim}}\left(d\left(p_{i}^{A}, p_{i}^{P}\right)\right)}{n}
$$

and

$$
d=\left(1-\frac{\sum_{i=1}^{n} \max \left(h\left(p_{i}^{A}\right), h\left(p_{i}^{P}\right)\right)}{n}\right) \cdot\left(1-\frac{\sum_{i=1}^{n} \mu_{\operatorname{Sim}}\left(d\left(p_{i}^{A}, p_{i}^{P}\right)\right)}{n}\right) .
$$

The hesitation $h$ in the $\operatorname{BSD}(s, d)$ is $h=1-s-d=\frac{\sum_{i=1}^{n} \max \left(h\left(p_{i}^{A}\right), h\left(p_{i}^{P}\right)\right)}{n} \in[0,1]$ and again $s+d=1-h \leq 1$.

\subsection{Interpreting the Results}

In a typical victim identification search, a PM 3D ear model is compared with a set of $m$ AM $3 \mathrm{D}$ ear models taken from a database with missing persons. Each of these comparisons results in a $\operatorname{BSD}\left(s_{i}, d_{i}\right), i=1, \ldots, m$, from which the hesitation $h_{i}=1-s_{i}-d_{i}$ about the result can be derived. Hence, the information provided from the comparison is the following:

1. $s_{i}(\in[0,1])$ : denotes how satisfied/convinced the method is about the matching of both ear models.

2. $d_{i}(\in[0,1])$ : denotes how dissatisfied/unconvinced the method is.

3. $h_{i}(\in[0,1])$ : expresses the overall hesitation about the comparison results (due to inadequate data quality).

In practice, forensic experts will be interested in the top-k matches for a given PM 3D ear model. For that purpose, the resulting BSDs $\left(s_{i}, d_{i}\right), i=1, \ldots, m$, have to be ranked. In the given context, the best ear matches are those where $s_{i}$ is as high as possible and $h_{i}$ is as low as possible. Therefore, considering that $h_{i}=1-s_{i}-d_{i}$, the ranking function

$$
\begin{aligned}
r:[0,1]^{2} & \rightarrow[0,1] \\
(s, d) & \mapsto \frac{s+(1-d)}{2}
\end{aligned}
$$

can be used. This function computes a single ranking value $r\left(\left(s_{i}, d_{i}\right)\right)$ for each $\operatorname{BSD}\left(s_{i}, d_{i}\right)$, which can then be used to rank order the comparison results and select the top-k among them. Other ranking functions are possible and discussed in [17].

Another option is to work with two threshold values $\delta_{s}, \delta_{h} \in[0,1]$. In such a case, only ear models for which the resulting $\operatorname{BSD}(s, d)$ satisfies $s \geq \delta_{s}$ and $h \leq \delta_{h}$ (or $1-s-d \leq \delta_{h}$ ) are kept in the comparison result. 


\section{Conclusions and Future Work}

In this paper, we described some theoretical aspects of a novel, bipolar approach for comparing 3D ear models. Soft computing techniques based on heterogeneous bipolar satisfaction degrees (BSDs) support explicitly coping with the hesitation that occurs when low(er) quality ear photos have to be compared with other ear photos (taken in different position, on a different time, ...). The use of BSDs allows to provide user with extra quantitative information about the overall hesitation on the comparison results (due to inadequate data quality).

The focus in the paper is on the ear recognition and decision processes of an ear identification approach. The presented technique departs from a 3D ear model that is obtained from ear detection, normalisation and enhancement processes. On this model, parts of low quality are annotated using a set of so-called hesitation spheres. From each 3D ear model a feature list is extracted. Feature lists are compared with each other by using a novel bipolar similarity measure, which provides quantitative information on the similarity of two ears and on the overall hesitation about (the quality of) the data involved in the comparison.

Up to now the method has only been tested on synthetically modified ear models. Experiments with models of real ears are necessary for parameter finetuning and validation purposes and are planned in the near future.

Acknowledgements. This work is supported by the Flemish Fund for Scientific Research.

\section{References}

1. Abate, A., Nappi, N., Riccio, D., Ricciardi, R.: Ear recognition by means of a rotation invariant descriptor. In: 18th IEEE International Conference on Pattern Recognition (ICPR), pp. 437-440 (2006)

2. Abaza, A., Ross, A., Hebert, C., Harrison, M.A., Nixon, M.S.: A Survey on Ear Biometrics. ACM Computing Surveys 45(2), article 22 (2013)

3. Abdel-Mottaleb, M., Zhou, J.: Human ear recognition from face profile images. In: Zhang, D., Jain, A.K. (eds.) ICB 2006. LNCS, vol. 3832, pp. 786-792. Springer, Heidelberg (2006)

4. Atanassov, K.: Intuitionistic fuzzy sets. Fuzzy Sets and Systems 20, 87-96 (1986)

5. Burge, M., Burger, W.: Ear biometrics. In: Jain, A., et al. (eds.) Biometrics: Personal Identification in Networked Society. Kluwer Academic Publishers (1998)

6. Cadavid, S., Abdel-Mottaleb, M.: 3D ear modeling and recognition from video sequences using shape from shading. In: 19th IEEE International Conference on Pattern Recognition (ICPR), pp. 1-4 (2008)

7. Chen, H., Bhanu, B.: Contour matching for 3D ear recognition. In: IEEE Workshops on Application of Computer Vision (WACV), pp. 123-128 (2005)

8. Choras, M., Choras, R.: Geometrical algorithms of ear contour shape representation and feature extraction. In: 6th IEEE International Conference on Intelligent Systems Design and Applications (ISDA), pp. 123-128 (2006)

9. Cummings, A.H., Nixon, M.S., Carter, J.N.: A Novel Ray Analogy for Enrolment of Ear Biometrics. In: 4th IEEE Conference on Biometrics: Theory, Applications and Systems, pp. 302-308 (2010) 
10. Dubois, D., Prade, H.: An introduction to bipolar representations of information and preference. International Journal of Intelligent Systems 23, 866-877 (2008)

11. Feng, J., Mu, Z.: Texture analysis for ear recognition using local feature descriptor and transform filter. In: SPIE (MIPPR 2009: Pattern Recognition and Computer Vision, vol. 7496, Session 1 (2009)

12. Gutierrez, L., Melin, P., Lopez, M.: Modular neural network integrator for human recognition from ear images. In: International Joint Conference on Neural Networks, pp. 1-5 (2010)

13. Iannarelli, A.: Ear Identification. Forensic Identification Series. Paramount Publishing Company, California (1989)

14. Islam, S., Bennamoun, M., Davies, R.: Fast and fully automatic ear detection using cascaded adaboost. In: IEEE Workshop on Applications of Computer Vision, pp. $1-6(2008)$

15. Kisku, D.R., Mehrotra, H., Gupta, P., Sing, J.K.: SIFT-Based ear recognition by fusion of detected key-points from color similarity slice regions. In: IEEE International Conference on Advances in Computational Tools for Engineering Applications (ACTEA), pp. 380-385 (2009)

16. Matthé, T., De Tré, G., Zadrozny, S., Kacprzyk, J., Bronselaer, A.: Bipolar Database Querying Using Bipolar Satisfaction Degrees. International Journal of Intelligent Systems 26(10), 890-910 (2011)

17. Matthé, T., De Tré, G.: Ranking of bipolar satisfaction degrees. In: Greco, S., Bouchon-Meunier, B., Coletti, G., Fedrizzi, M., Matarazzo, B., Yager, R.R. (eds.) IPMU 2012, Part II. CCIS, vol. 298, pp. 461-470. Springer, Heidelberg (2012)

18. Nanni, L., Lumini, A.: A supervised method to discriminate between impostors and genuine in biometry. Expert Systems with Applications 36(7), 10401-10407 (2009)

19. Nielandt, J., Bronselaer, A., Matthé, T., De Tré, G.: Bipolarity in ear biometrics. In: European Society for Fuzzy Logic and Technology Conference and les rencontres francophones sur la Logique Floue et ses Applications (EUSFLAT/LFA), pp. 409$415(2011)$

20. Passalis, G., Kakadiaris, I., Theoharis, T., Toderici, G., Papaioannou, T.: Towards fast 3D ear recognition for real-life biometric applications. In: IEEE Conference on Advanced Video and Signal Based Surveillance, pp. 39-44 (2007)

21. Sánchez, D., Melin, P.: Optimization of modular granular neural networks using hierarchical genetic algorithms for human recognition using the ear biometric measure. Engineering Applications of Artificial Intelligence 27, 41-56 (2014)

22. Xie, Z., Mu, Z.: Ear recognition using lle and idlle algorithm. In: 19th IEEE International Conference on Pattern Recognition (ICPR), pp. 1-4 (2008)

23. Yan, P., Bowyer, K., Chang, K.: ICP-Based approaches for 3D ear recognition. In: SPIE (Biometric Technology for Human Identification II), pp. 282-291 (2005)

24. Yan, P., Bowyer, K.: Biometric recognition using 3D ear shape. IEEE Transactions on Pattern Analysis and Machine Intelligence 29(8), 1297-1308 (2007)

25. Yuizono, T., Wang, Y., Satoh, K., Nakayama, S.: Study on individual recognition for ear images by using genetic local search. In: IEEE Congress on Evolutionary Computation (CEC), pp. 237-242 (2002)

26. Zhang, H., Mu, Z., Qu, W., Liu, L., Zhang, C.: A novel approach for ear recognition based on ICA and RBF network. In: 4th IEEE International Conference on Machine Learning and Cybernetics, pp. 4511-4515 (2005) 